\title{
IASOS DI CARIA E IL RILANCIO DEL SUO PORTO IN ETÀ BIZANTINA: IL RUOLO DEL MARMO
}

\section{FEDE BERTI, DIEGO PEIRANO}

UDC: $7 \cdot 033 \cdot 2: 711 \cdot 453 \cdot 4(38)$ 691.214 .8

Original scientific paper

Manuscript received: 15. 10. 2015.

Revised manuscript accepted: 16. 03. 2016.

DOI: 10.1484/J.HAM.5111341
F. Berti

Via Bagaro 6, 44121 Ferrara

Italia

D. Peirano

Via Chiesa della Salute 52, 10147 Torino Italia

Placed inside one of the deep inlets indenting the Carian Coast, Iasos knew a period of economic prosperity in Justinian times, thanks to the increased demand of the polychrome marble quarried locally. At that time the marble, extracted from the nearby reliefs, was used mainly in revetment slabs. These were used in open book compositions which embellished many imperial buildings. The marbles were sawn into slabs in the quadriporticus of the mausoleum of the $2^{\text {nd }}$ century A.D. placed nearby the East harbor. In that building, placed close to the aqueduct, were found numerous marble blocks, showing saw traces, abandoned over thick emery layers. The East harbor seems therefore the starting place for the marble artifacts diffusion, ascertained on eastern Mediterranean shores and Italy. At the time probably the quarries were imperial properties; so it is likely that here worked also Constantinopolitan stonemasons. These exchanges with Constantinople brought in Iasos the coloristic taste typical of the period: this is attested by the agora basilica, where, in the Justinian age, different kinds of marble were used, some maybe imported from the capital.

Keywords: Iasos, marmor iassense, use of marble, harbors

"La sala era di forma circolare...Il pavimento a disegni geometrici era composto a rombi di cipollino rosso intrecciati a lastre compatte di porfido e di marmo di Aquitania”.

(L. Malerba, Il fuoco greco, Mondadori 2000, p. 10)

Iasos, in Caria, bilanciava la limitata estensione della sua chora con la vastità del mare antistante. La penisola sulla quale sorse si trova sul fondo del golfo che per Tucidide (VIII,

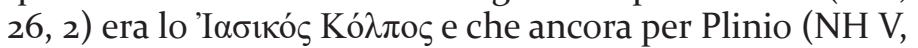
29,107) era il sinus Iasius (fig. 1).
Voce importante, accanto alla pesca, nell'economia locale fu certamente il marmor iassense.

Le vene della pietra si estendono nell'entroterra della penisola da nord verso est ${ }^{1}$, salendo quasi dal fondo valle fino al valico soprastante il "mare piccolo", dove, dall'ampio fronte

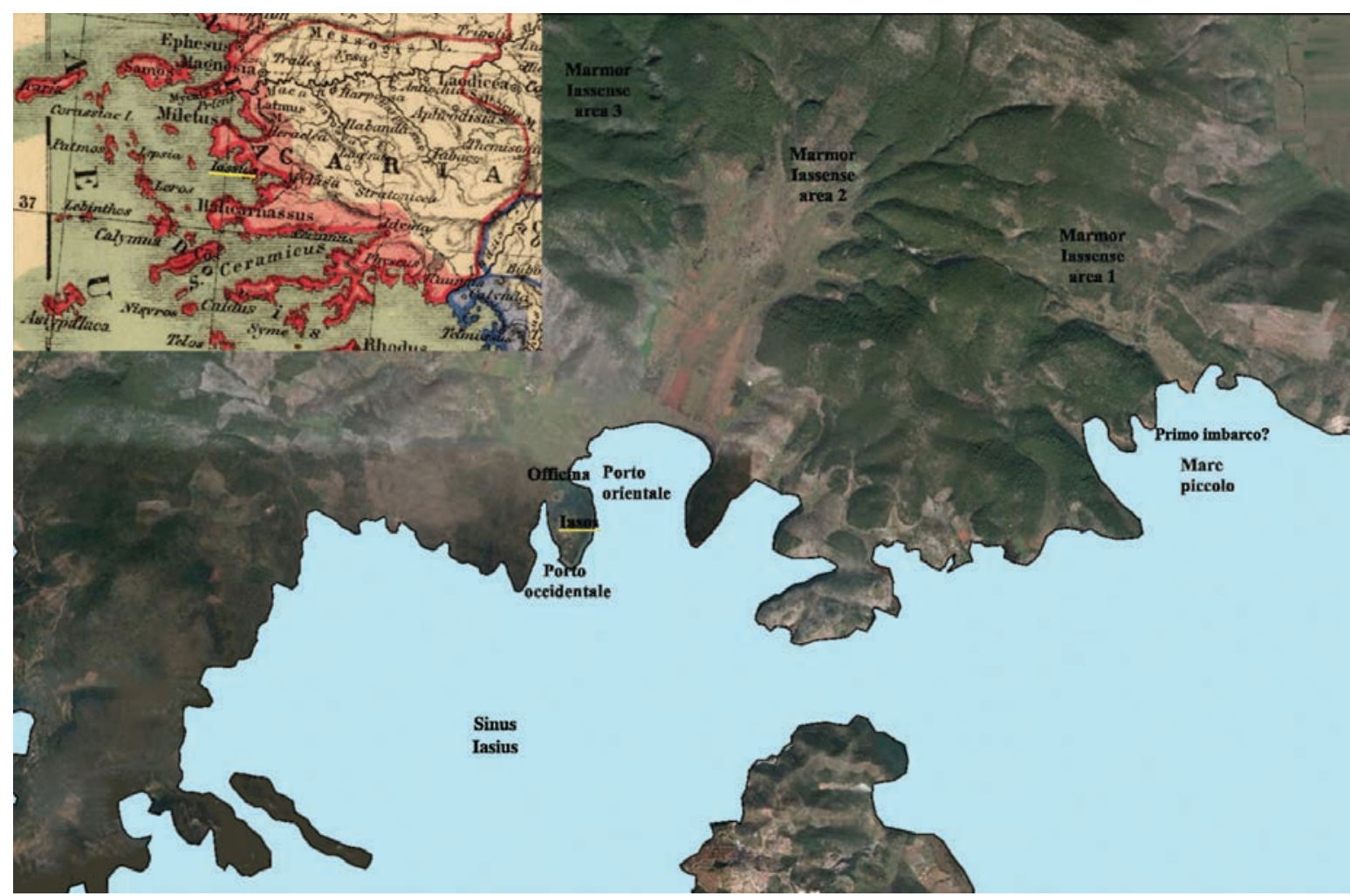

Fig. 1: in alto a sinistra una mappa della Caria (dal Kiepert); a destra una foto satellitare di Iasos e del suo territorio con evidenziate alcune aree di estrazione, trasporto e lavorazione del marmor iassense.

${ }^{1}$ Il marmo affiora anche verso Akbük (D. MONNA, P. PENSABENE, Marmi dell'Asia Minore, Roma, 1977, p. 109 ss.), tuttavia le cave antiche si trovano nella chora di Iasos. 


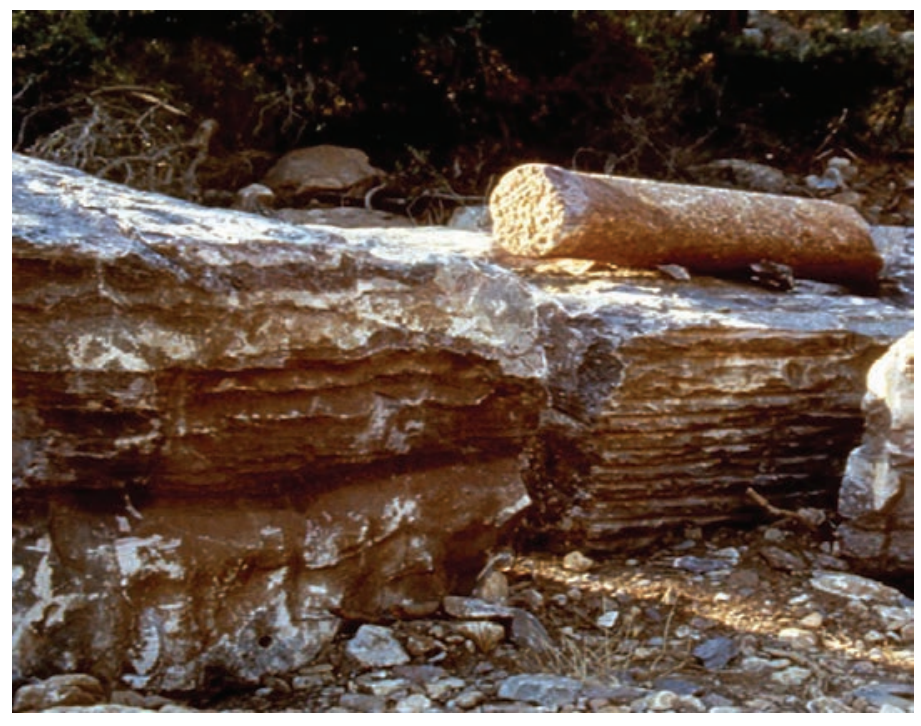

Fig. 2: una colonna sbozzata (foto Daniela Baldoni)

di cava, si prelevavano sia il cipollino, sia il monocromo. La breccia si traeva da altri settori ${ }^{2}$. Nel medio e tardo Bronzo da questo marmo d'intenso colore rosso, chiazzato o striato di bianco (breccia e cipollino) oppure violaceo (il monocromo, talora disseminato di minuscoli inclusi bianchi), si ricavavano lampade, piattelli, fusaiole3. Nel IV secolo a.C. e in periodo ellenistico, nella chora, si faceva ricorso allo stesso per erigere torri e costruire terrazzamenti e edifici. Del più imponente di questi ultimi complessi, che ha restituito materiali di II secolo a.C., sul Karaoğlan Deresi, restano anche elementi architettonico-strutturali quali un piccolo capitello dorico e un blocco del paramento murario (fig. 2) ${ }^{4}$. Per ciò che riguarda lo sfruttamento delle cave (questione di cui assai poco si sa), si riteneva che l'esportazione del lavorato fosse iniziata in periodo flavio, tuttavia la colonna di cipollino rosso della cattedrale di Bari dedicata Soli sacr(um) dall'augustale $Q$. Terentius Q. l./ Anteros attesta che commercio e (tanto più) estrazione risalgono a età precedente ${ }^{5}$. Il dato trova conforto nel ruolo di stazione doganale svolto dalla città, il cui nome è presente nel Monumentum Ephesenum, testo che -come è noto- riprende nel 62 d.C. un documento del 75 a.C. ${ }^{6}$.

In città il marmo iassense, qualunque ne fosse il tipo, è raro prima del VI secolo d.C.7; pertanto, l'importazione di marmi bianco/grigi da altre cave della regione (si pensi, ad esempio, al marmo di Mylasa dei colonnati dell'agorà imperiale di età adrianea-antonina) non sembra riconducibile a meccanismi territoriali di scambio poiché il marmor iassense, nei secoli centrali dell'impero, già si muove in prevalenza verso paesi d'oltremare. A partire dal VI secolo esso si fa più frequente tanto nei mosaici e nei sectilia pavimentali degli edifici di culto cristiano, come nei loro arredi, dove figurano colonne in breccia e in cipollino di piccola e media dimensione.

E' comunque nell'esportazione che, a partire dall'età proto-bizantina, si misura il cambiamento: il "cipollino rosso" va ad abbellire importanti complessi architettonici a Costantinopoli, Ravenna e altrove; la policromia della pietra, sapientemente rafforzata da una messa in opera "a spina" (nel caso di rivestimenti parietali), colpisce la vista ed è celebrata dagli scrittori di corte ${ }^{8}$. Come dire che da Iasos, statio doganale dal I secolo a. C., è dal VI che, via mare, si muove con maggiore intensità un flusso di materiali divenuti "di moda".

Tale traffico deve aver rivitalizzato la città, come sembrano suggerire alcuni tra i ritrovamenti urbani e i dati sulla diffusione del marmo.

${ }^{2}$ F. BERTI, Marmo Iasio, I, in Corsi di Cultura e Arte Bizantina 48, 1998, p. 61-74 (manufatti di marmo iasio nella città); A. ANDREOLI, F. BERTI, L. LAZZARINI, R. PIEROBON BENOIT, New contribution on marmor iassense, in L. LAZZARINI (ed.), Interdisciplinary studies on ancient stone, ASMOSIA VI, Proceedings of the Sixth International Conference of the Association for the Study of Marble and Other Stones in Antiquity (Venice, June 15-18 200o), Padova, 2002, p. 13-18 e R. PIEROBON BENOIT, Paralypros chora, in Iasos e la Caria. Nuovi studi e ricerche, in La Parola del Passato, 341-345, 2005, p. 232 s. e L. LAZZARINI, S. CANCELLIERE, R. PIEROBON BENOIT, Il marmo di Iaso: cave, uso, caratterizzazione e indagini archeometriche, ibidem, p. $320-331$ (le cave); M. BRUNO, Quarry blocks in marmor iassense from the Balik Pazari at Iasos (Turkey), in A. GUTIERREZ GARCIA, M. PILAR LAPUENTE, I. RODÂ (eds.), Interdisciplinary Studies on Ancient Stone. Proceedings of the IX ASMOSIA Conference (Tarragona 8-13th June 2009), Tarragona, 2012, p. 706- 713 (i ritrovamenti nel Balık Pazarı).

${ }^{3}$ Alcuni di questi oggetti in N. MOMIGLIANO et alii, Bronze Age Carian Iasos. Structures and Finds from the Area of the Roman Agora (c. $3000-1500$ BC), Roma, 2012, p. 107, 151, 158, 162, fig. 168.

${ }^{4}$ Sull'edificio del Karaoğlan Deresi F. BERTI, The work of the Italian Archaeological Mission at Iasos, 1999, in 22. Kazi Sonuçları Toplantisi (Izmir, 22-26 Mayis 2000) Ankara 2001, 2, p. 121 S.

${ }^{5}$ Difficilmente posteriore, in base al ductus epigrafico, ai primi decenni del I secolo d. C.: M. SILVESTRINI, Una dedica al Sole nella cattedrale di Bari, in M. SILVESTRINI, T. SPAGNUOLO VIGORITA, G. VOLPE (a cura di), Studi in onore di Francesco Grelle, Bari, 2006, p. 267-279. Di età flavia scriveva R. GNOLI, Marmora Romana, Roma, 1988, p. 210-211.

${ }^{6}$ D. G. MEROLA, Autonomia locale, governo imperiale. Fiscalità e amministrazione nelle province asiane, Bari, 2001, passim; G. D. ROWE, The Elaboration and Diffusion of the Text of the Monumentum Ephesenum, in M. COTTIER et alii, (eds.) The Customs Law of Asia, Oxford, 2008, p. 236-250.

$7 \mathrm{Il}$ fenomeno è condiviso con altri centri dotati di aree estrattive: sull'argomento J. C. FANT, Ideology, gift, and trade: a distribution model for the Roman imperial marbles, in W. V. HARRIS (ed.), The Inscribed Economy. Production and distribution in the Roman empire in the light of instrumentum domesticum, in Journal of Roman Archaeology, suppl. series 6, 1993, p. 153 s. In età augustea la breccia rossa fa una timida apparizione nell'opus sectile della stoà orientale dell'agorà, in seguito nel pavimento del bouleuterion. Nell'edificio sono in opera anche blocchi di "monocromo", al quale si ricorse per le campiture rosse dei tessellati di II secolo d. C. della "casa dei mosaici" e del Balık Pazarı. Il marmo locale è invece quasi assente nel deposito di lastre lisce e modanate, di cornici e di piastrelle sigillato da un incendio nell'avanzato III secolo ritrovato in una delle botteghe della stoà occidentale dell'agorà: cfr. F. BERTI, F. DELRIEUX, Un luogo di vendita dell'età medio-imperiale nell'agora di Iasos, in V. CHANKOWSKI, P. KARVONIS (dir.), Tout vendre, tout acheter. Structures et équipements des marchés antiques, Actes du colloque d'Athènes (16-19 juin 2009), Paris, 2012, p. 105- 118. Le vicende edilizie del bouleuterion si pongono tra il I e il II secolo d. C.: R. PARAPETTI, Il bouleuterion: aspetti architettonici e decorativi, in Studi su Iasos di Caria, in Bollettino d'Arte, suppl. al n. 31-32, 1985, p. 105- 136.

${ }^{8}$ Il gusto per i marmi policromi allora e in seguito coltivato traspare anche dalle raffigurazioni su codici e manoscritti: cfr. A. PARIBENI, L'uso e il gusto del marmo in età bizantina attraverso le descrizioni e le rappresentazioni antiche, in E. DOLCI (a cura di), Il marmo nella cultura romana, Carrara 1989, figg. 7 e 15 (codice athonita e manoscritto armeno), dove le specchiature paiono di cipollino iasio. Fa riferimento alla stessa pietra, indicandone alcune ricorrenze nelle stesure musive pavimentali che la imitano, D. MICHAELIDIS, Some aspects of marble imitation in mosaic, in P. PENSABENE (a cura di), Marmi antichi. Problemi d'impiego, di restauro e d'identificazione, in Studi Miscellanei, 26, 1985, p. 159, 161 e s. e tavv. 5,1 e 6,3. 

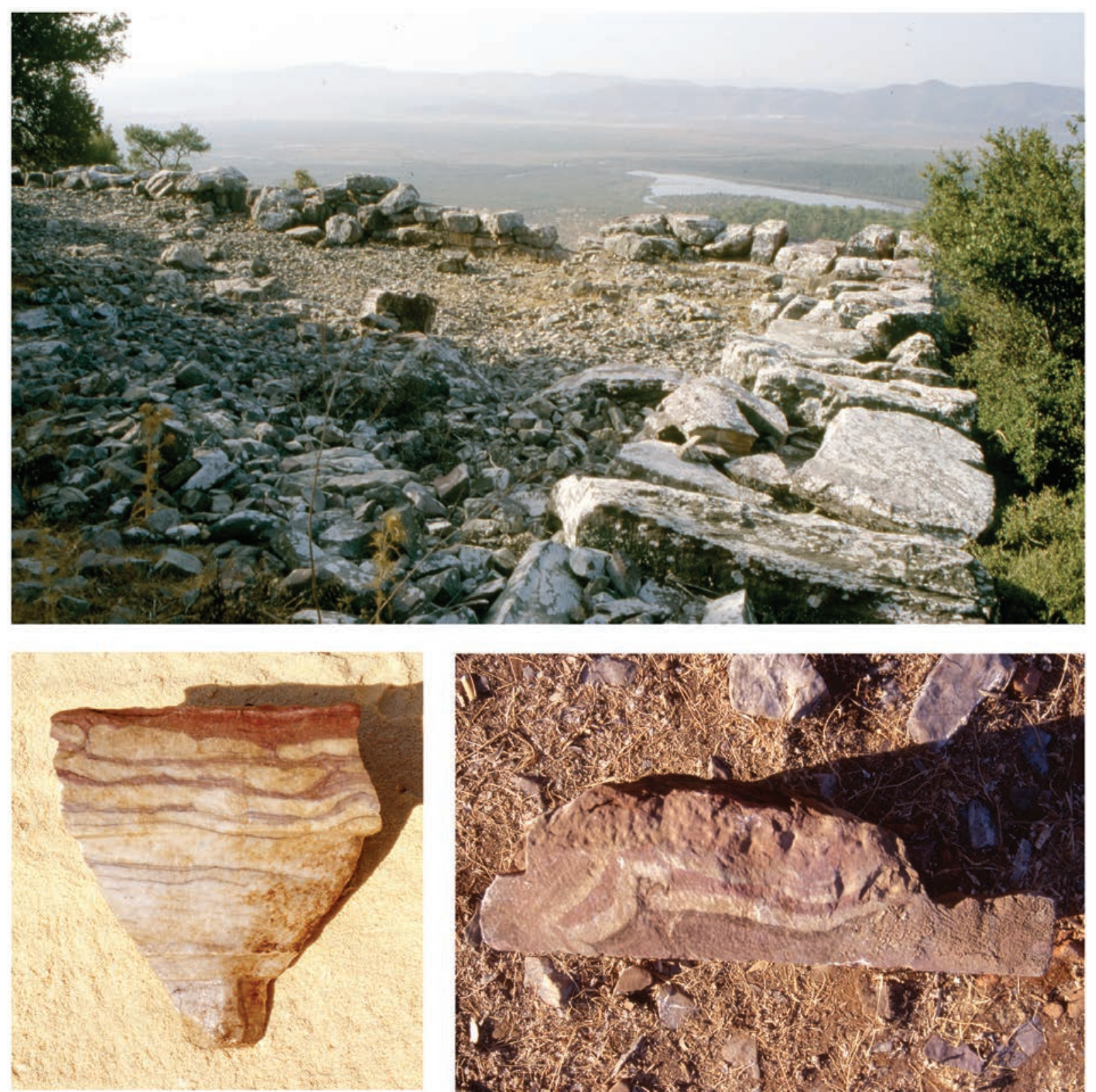

Fig. 3: in alto il terrazzamento del Karaoğlan Deresi che sovrasta il 'mare piccolo', in basso a sinistra il frammento di un piccolo capitello dorico, a destra un blocco del paramento murario del vicino edificio (foto Daniela Baldoni)

Se la lavorazione di alcuni manufatti avveniva in cava (fig. 2), dove sussistevano sia spiazzi di stoccaggio, sia edifici per lavoranti e addetti al controllo, il polo extraurbano della lavorazione del marmo era ubicato, a Iasos, nel complesso funerario di età imperiale denominato "mercato del pesce" (Balık Pazarı). Il grande edificio (figg. 4/1 e 5) si compone di un quadriportico e di un tempio corinzio al centro di una corte, con sepolture nel podio del tempio e negli annessi ${ }^{9}$.
Qui sono stati ritrovati, sopra uno strato di sabbia di quarzo, 114 blocchi di "cipollino iasio", 44, con segni di taglio ${ }^{10}$ (fig. 6), pochi frammisti al crollo del tempio, i restanti nei lati settentrionale e orientale del quadriportico. Il mausoleo fiancheggia l'acquedotto (figg. 4/1-2) e si trova a breve distanza dal porto orientale: il marmo vi era segato con la medesima tecnica usata nei secoli VI-VII a Efeso ${ }^{11}$ e Gerasa ${ }^{12}$ e prima, nel III, a Hierapolis ${ }^{13}$, e da lì era avviato all'imbarco.

\footnotetext{
9 R. PARAPETTI, Anastilosi grafica del monumento funerario del Balik Pazari di Iasos, in D. BALDONI, F. BERTI, M. GIUMAN (a cura di), Iasos e il suo territorio, Roma, 2013, p. 161- 172.

${ }^{10}$ M. BRUNO, op. cit. (n. 2), p. 706- 713.

"F. MANGARTZ, Die byzantinische Steinsäge von Ephesos, Regensburg 2010: tra i 400 pezzi ritrovati in situ e schedati, prevalentemente di marmo bianco, soltanto 6 (i nr. 114, 125, 142, 146, 156, 377) sono di marmo rosso. Ci si chiede se non possa trattarsi di marmo iasio, come sembra che sia il nr. 95, il solo fotografato (tav. 19.1: weisser Marmor rot geädert).

${ }^{12}$ J. SEIGNE, Water-powered Stone Saws in Late Antiquity. First Step on the Way to Industrialisation?, in G. WIPLINGER (ed.), Cura Aquarum in Ephesus. Proceedings of the Twelfth International Congress on the History of Water Management and Hydraulic Engineering in the Mediterranean Region (EphesusSelçuk 2-10 October 2004), Dudley-Leuven, 2006, p. 371- 378.

${ }_{13}$ T. RITTI, K. GREWE, P. KESSENER, A relief of a water-powered stone saw mill on a sarcophagus at Hierapolis and its implications, in Journal of Roman Archaeology 20, 2007, p. 139-163: sarcofago di M. Aur. Ammianus con la prima raffigurazione di una sega azionata da acqua. Blocchi con tagli ravvicinati sono segnalati un po' ovunque: qualche esempio in T. KOZELJ, M. WURCH-KOZELJ, Use of saw in roman and proto-byzantine period on the island of
} 


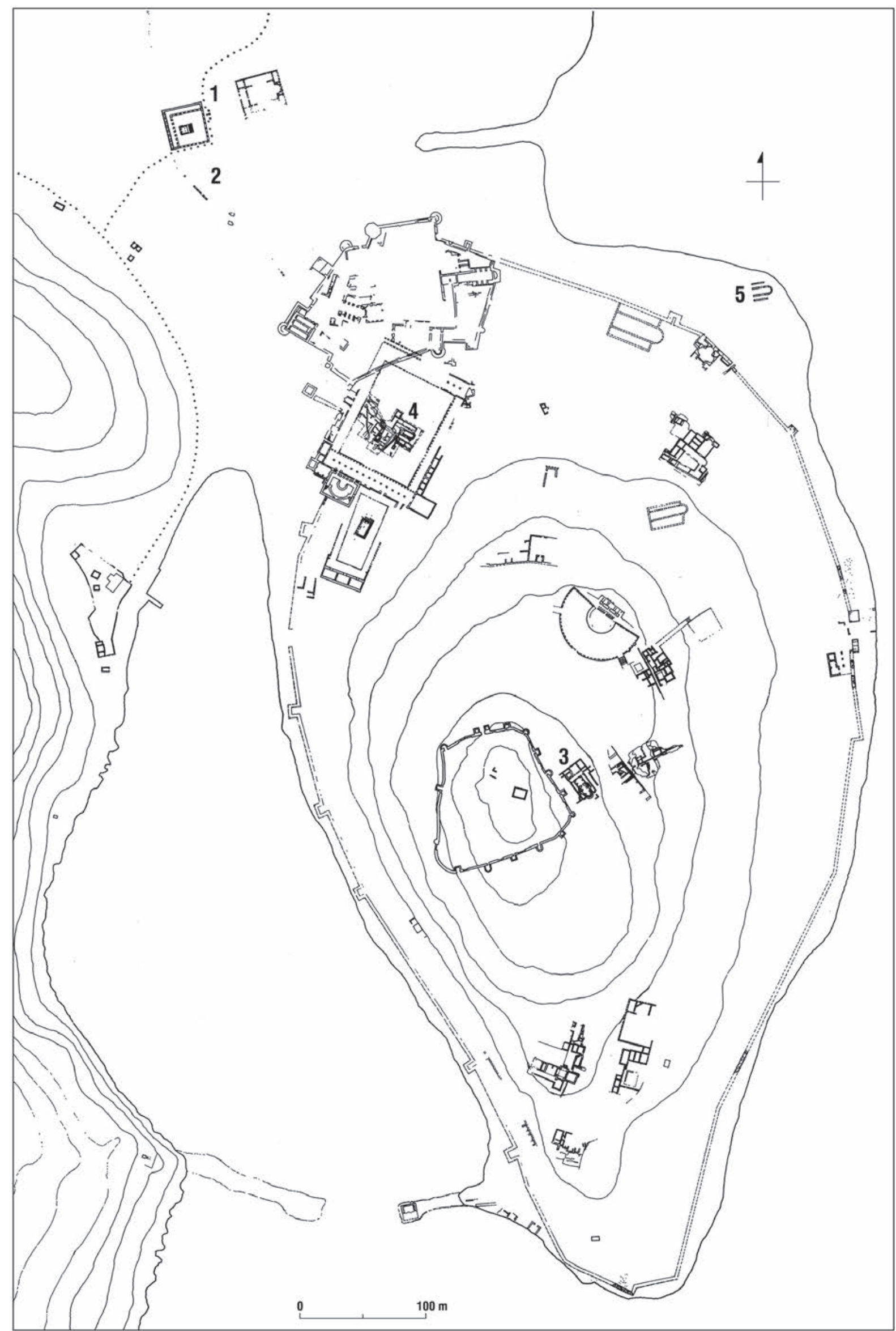

Fig. 4: mappa della penisola di Iasos con evidenziati gli edifici citati nel presente contributo

Thasos, in L. LAZZARINI (ed.), Interdisciplinary Studies ....op. cit. (n. 2), p. 715 s., fig. 11c. Generalità in M. L. BRUTO, C. VANNICOLA, Strumenti e tecniche di lavorazione dei marmi antichi, in Archeologia Classica 42, 1990, p. 287-324 e M. BRUNO, Considerazioni sulle cave, sui metodi di estrazione, di lavorazione e sui trasporti, in M. DE NUCCIO, L. UNGARO (a cura di), I marmi colorati della Roma imperiale, Venezia, 2002 , p. 188 ss. 

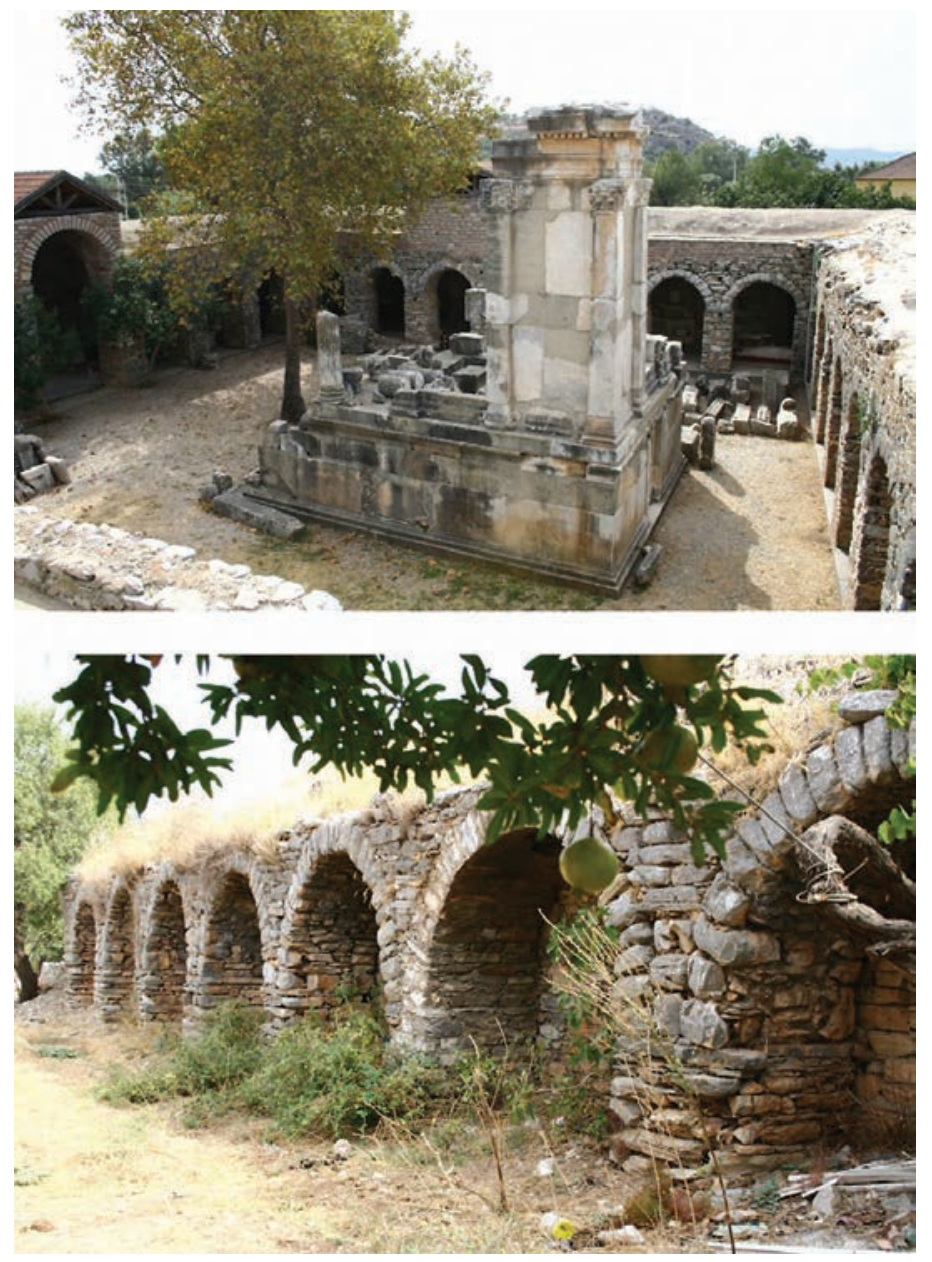

Fig. 5: sopra, vista dall'alto della tomba nota come 'Balık Pazari'; sotto l'acquedotto a cui si appoggia il lato ovest del quadriportico (foto Maurizio Molinari)

La dislocazione dell'officina e la presenza del cospicuo letto di smeriglio richiamano alla mente l'editto emanato nel 146/147 d. C. dal proconsole Lucio Antonio Albo, che proibiva ai trasportatori di legnami e di marmi di scaricare i residui delle lavorazioni nel porto di Efeso che, evidentemente, già stava interrandosi ${ }^{14}$.

Che il cantiere iasio risalga al periodo bizantino lo confermano le croci, accompagnate da lettere singole e non, incise su alcuni blocchi anche dopo l'estrazione.

Al commento che, nell'edizione, accompagna tali sigle si aggiungono ora alcune annotazioni, utili per ampliare la casistica relativa a una materia che rimane decisamente criptica ${ }^{15}$ :

- il segno Bruno 2012, fig. 10/1-2 è simile al segno Deichmann 1976, fig. 51;

- il segno Bruno 2012, fig. 10,/9 è simile al segno Deichmann 1976, tav. 4 (su verde di Tessaglia, rosso di Synnada e marmo proconnesio; nel nostro caso sarebbe semplificato);

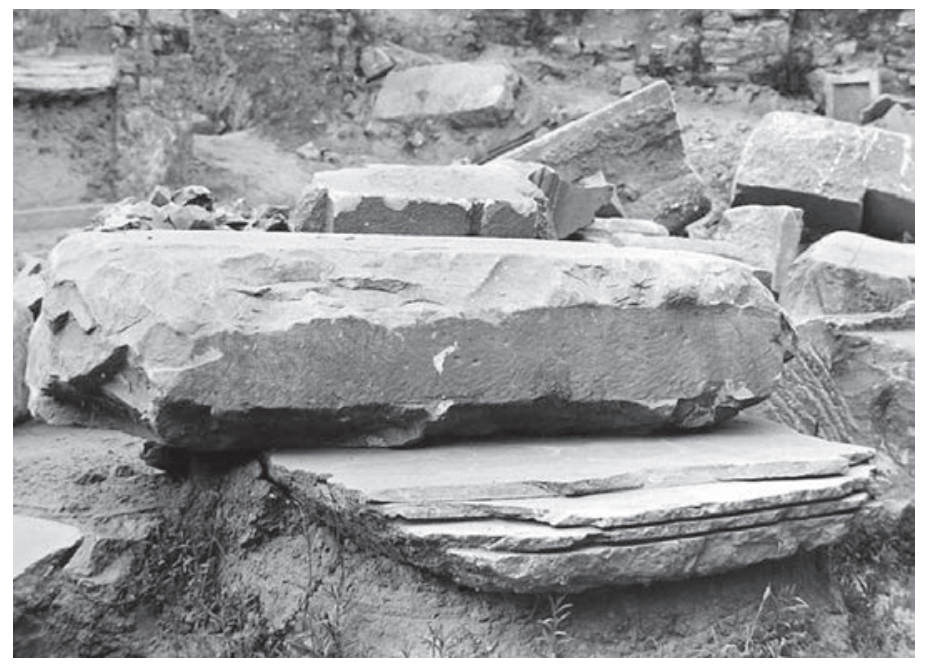

Fig. 6: una foto degli scavi degli anni '60 del 'Balik Pazari' con uno dei blocchi con tracce di segagione (scavi Levi, Archivio SAIA)

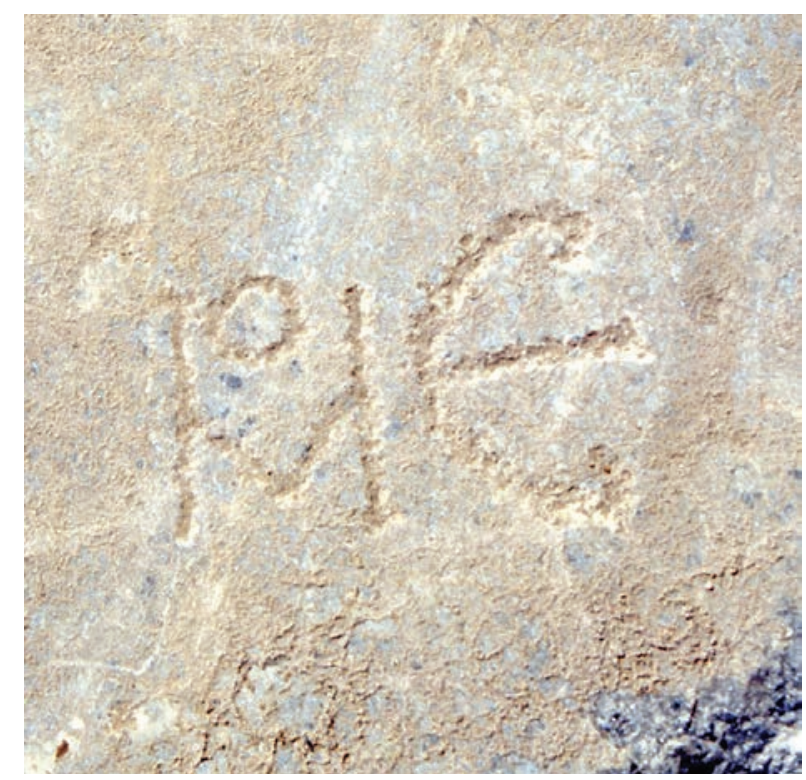

Fig. 7: sigla ritrovata su uno dei blocchi del 'Balık Pazari' (foto Daniela Baldoni)

- sul blocco Bruno 2012, fig.10/10 figurano due gruppi di segni. Quello in alto a sinistra è noto anche a Deichmann 1976, tav. 4a. Il nesso di due lettere con cui inizia quello in basso a destra, in accordo con Bruno, pare costituito da un $\Pi$ sormontato lateralmente da un omicron: sarebbe quindi l'abbreviazione di $\pi$ ov́ presente nella versione greca del capitolo sui marmi dell'Editto di Diocleziano e nelle cave di Mons Claudianus ${ }^{16}$. Il gruppo di questi graffiti incisi sui blocchi del cantiere si arricchisce inoltre di una nuova sigla di tre lettere (fig. 7), non meno sibillina delle precedenti ${ }^{17}$.

I dati dello scavo (non esteso) non chiariscono come si vorrebbe la successione degli eventi sopravvenuti nel tempo

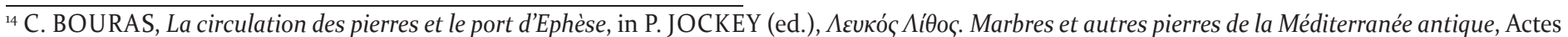
du VIII colloque international de l'Association ASMOSIA (Aix-en-Provence, 12-18 juin 2006), Aix-en-Provence 2009, p. 495-508. Sul porto di Efeso cfr. inoltre M. SPANU, Per lo studio del Porto di Efeso, in Daidalos 3, 2001, p. 215- 248.

${ }^{15}$ M. BRUNO, Quarry blocks, op. cit. (n. 2), p. 710 s. e fig. 10 e F. W. Deichmann, Ravenna. Hauptstadt des spätantiken Abendlandes, 2, Wiesbaden, 1976, p. $215-216$.

${ }^{16}$ S. CORCORAN, J. DE LAINE, The unit measurement of marble in Diocletian's Prices Edict, in Journal of Roman Archaeology 7, 1994, p. 264-5.

${ }^{17}$ Qualcosa di simile (un monogramma con my, omicron ed epsilon non lunato) si vede su blocchi dell'acquedotto di Istanbul, nel tratto di Kumarlıdere: J. BARDILL, The Mason's Marks, in J. CROW, J. BARDILL, R. BAYLISS (eds.), The Water Supply of Byzantine Costantinople (Journal of Roman Studies, Monograph 11), 2008, p. 202-3. E' contraddistinto da segni/numeri (ma ci si chiede se siano antichi) anche un blocco di cipollino rosso affiorante in un settore non scavato dell'agorà, a lasos. 
all'interno e all'esterno del quadriportico, a fronte del quale, a oriente, si trovano una delle basiliche cristiane (forse la più antica) di Iasos e un sepolcreto. Se una ridotta trincea aperta a ridosso del muro di spalla del portico ha riportato alla luce 16 fosse terragne con inumati privi di oggetti di corredo, nessuna tomba fu ritrovata durante lo scavo del portico, sopra o tra i blocchi. Da tale situazione, allo stato delle cose, si può ricavare che necropoli e, probabilmente, chiesa oltrepassarono nel tempo la durata del cantiere e che in periodo proto-bizantino l'acquedotto funzionava, tanto che la sua portata non era ridotta dal prelievo (seppur intermittente) dell'acqua per le segagioni.

Non sappiamo nemmeno quando iniziarono le lavorazioni e per quanto tempo durarono: fu per la scarsa qualità del marmo che i 114 blocchi furono tralasciati o vi fu qualche altra ragione? In casi simili si suppone che l'attività non sia stata interrotta repentinamente, né dallo spessore del letto di sabbia quarzifera, che è veramente notevole, si ricavano altre informazioni ${ }^{18}$.

Anche circa la provenienza dei blocchi si vorrebbe conoscere qualcosa di più: l'estrazione era avvenuta nella cava più vicina alla città sita in una gola (con un aggravio del costo e dei tempi del trasporto affidato ad animali) oppure nel più ampio ma più lontano fronte, sul Karaoğlan Deresi (fig. 1, aree 1 e 3). In tale caso, la via di lizza individuata nel corso delle ricognizioni nella chora consentiva la discesa del materiale fino al "mare piccolo", l'imbarco e il trasporto" ${ }^{19}$; dal porto orientale della penisola esso sarebbe poi ripartito, una volta che fosse o no stato lavorato.

La gerarchia esistente tra i porti faceva sì che anche quelli nei pressi delle cave, a volte piccoli ma molto attivi, fossero compresi in circuiti che facevano capo a porti più grandi e importanti, di raccolta e di ridistribuzione.
Comunque sia, il viaggio del nostro marmo proseguiva probabilmente per Efeso, l'emporion più importante d'Asia Minore (Strabo, XV,I,25), essendo forse Efeso la prima tappa verso (ad esempio) Costantinopoli.

L'elevato numero di relitti con marmo lavorato e semi-lavorato, che costituiva un 'complemento' rispetto a quanto era già in stiva e che veniva caricato a bordo anche in navigazione, getta luce sui mezzi di trasporto (le "navi lapidarie") e testimonianze particolarmente vivide al riguardo sono $\mathrm{i}$ relitti di La Mirande, di Torre Sgarlata, di Porto Nuovo e di Punta Scifo D ${ }^{20}$.

Se il mare era la principale via di diffusione del marmor iassense, che, come si è accennato, è scarsamente attestato nell'interno della regione e nelle aree limitrofe ${ }^{21}$, il mare ne rendeva economica l'estrazione. La mappa dei ritrovamenti, disegnando due cerchi grossomodo concentrici (il primo corrispondente al mare Egeo, il secondo al Mediterraneo centro-orientale), mostra come essi siano quasi esclusivamente attestati lungo le coste.

Il punto più alto della fortuna del marmo iasio coincise, lo ripetiamo, con il periodo proto-bizantino: allora cominciò a essere usato in varie costruzioni religiose e la sua policromia andò a ravvivare importanti complessi architettonici, nella capitale e altrove.

Il marmo di Iasos compare a Bosra nella chiesa dei SS. Sergio, Bacco e Leonzio, dedicata nel $512-513^{22}$. Nella Costantinopoli del VI secolo è attestato per la prima volta nella chiesa di S. Polieucto costruita tra il 524 e il 527, dove figurava in lastre di rivestimento e sectilia ${ }^{23}$. Nella descrizione di Santa Sofia, Paolo Silenziario menziona il marmo iasio con altri 12 tipi di marmi tratti da cave tutte -presumibilmente- di proprietà imperiale.

Descript. S. Sophiae 594-597:

${ }_{18}$ Per marmi residuali in aree di stoccaggio cfr. C. BARSANTI, A. GUIGLIA, A. PARIBENI, Le officine dell'imperatore: marmora byzantina, in A. C. QUINTAVALLE (a cura di), Medioevo: le officine, Atti del Convegno internazionale di studi (Parma 22-27 settembre 2009), Milano, 2010, nota 8. Per il porto di Efeso cfr. nota 15. Del tempo stimato necessario per il taglio del marmo (nelle variabili derivanti dai tipi di pietra) scrive B. KIILERICH, The opus sectile from Porta Marina at Ostia and the aesthetics interior decoration, in I. JACOBS (ed.), Production and Prosperity in the Theodosian Period, in Interdisciplinary Studies in Ancient Culture and Religion 14, p. 176.

${ }^{19}$ R. PIEROBON BENOIT, Il territorio di Iasos: nuove ricerche (2006-2008), in L. KARLSSON, S. CARLSSON (eds.), Labraunda and Karia. Proceedings of the International Symposium Commemorating Sixty Years of Swedish Archaeological Work in Labraunda (Stockholm, November 20-21th, 2008), in Boreas 32, 2011, p. 411 ss. Sul "mare piccolo" (o "mare interno") G. TRAINA, Ricognizione del settore Sari Cay. Considerazioni preliminari, in SINUS IASIUS I. Il territorio di Iasos: ricognizioni archeologiche 1988- 1989, in Annali della Scuola Normale Superiore di Pisa, s. III, 23/3-4, 1993 , p. 966 ss.

${ }_{20}$ B. RUSSELL, Lapis transmarinus: stone-carrying ships and the maritime distribution of stone in the Roman empire, in D. ROBINSON, A. WILSON (eds.), Maritime Archaeology and Ancient Trade in the Mediterranean, Oxford 2011, p. 139-155; che il marmo destinato a essere segato viaggiasse in blocchi e richiedesse, una volta arrivato, attrezzature e manodopera per lavorarlo non era, evidentemente, l'unica soluzione. Relitti con lastre marmoree, di VI secolo e oltre (Altinkum, Capo Bianco, Marzameni II, Kizilburun, Ekinlik Adasi, Dor 2001/1), in E. F. CASTAGNINO BERLINGHIERI, A. PARIBENI, Byzantine Merchant Ships and Marble Trade, Skyllis 2011, p. 64-75. Per le naves lapidariae P. A. GIANFROTTA, $\Sigma \mu \varepsilon \rho ı \varepsilon \varsigma$ : depositi portuali, marmi di cava e navi, in Orizzonti. Rassegna di archeologia 9, 2008, in particolare p. 86 ss.; C. BELTRAME, V. VITTORIO, Roman Ships Carrying Marble: Were These Vessels in Some Way Special ?, in N. GÜNSENIN (ed.), Between Continents. Proceedings of the Twelfth Symposium on Boat and Ships Archaeology (Istanbul 2009), Istanbul 2012, p. 141-148; S. MEDAGLIA, C. BELTRAME, L. LAZZARINI, La navis marmorum di età romana 'Punta scifo D' (Crotone). Risultati preliminari della prima campagna di indagini subacquee, in Rivista di Archeologia 37, 2013, p. 137- 165.

${ }^{21}$ Per Labraunda J. BLID, Felicium Temporum Reparatio. Labraunda in Late Antiquity (c. AD 300-60o), Stockholm 2012, p. 58, fig. 45; per Cnidos A. B. YALCIN, Alcune osservazioni sul decoro scultoreo e musivo delle chiese protobizantine di Cnido in Caria, in Bisanzio el'Occidente: arte, archeologia, storia. Studi in onore di Fernanda de Maffei, Roma 1996, p. 122, fig. 20; per Sinuri (santuario di alta antichità prossimo anch'esso ad affioramenti del marmo rosso, dove, come nella chora di Iasos, era usato per costruzioni e come supporto di iscrizioni) L. ROBERT, Le sanctuaire de Sinuri près de Mylasa, I, Paris 1945, p. 14, tav. VIII; per Xanthos M. G. FROIDEVAUX, M. P. RAYNAUD, Les sols en opus sectile et leur contexte architectural dans la Basilique épiscopale de Xanthos, in Mélanges Jean-Pierre Sodini, Paris 2005 (Travaux et Mémoires,15), p. 145; per Efeso M. BRUNO, Quarry blocks, op. cit. (n. 2), p. 706 (trapezoforo in marmo iasio).

${ }^{22}$ N. MASTURZO, Architettura siriana pre-giustinianea: la chiesa dei SS. Sergio, Bacco e Leonzio. Problemi interpretativi e di conservazione, in Corsi di Cultura e Arte Bizantina 41, Ravenna, Costantinopoli, Vicino Oriente. In memoria del prof. Friedrich Wilhelm Deichmann, (Ravenna 12-16 settembre 1994), Ravenna 1995, p. 378: rivestimenti parietali.

${ }^{23}$ R. M. HARRISON, Monumentalità e urbanistica nella città, in A. GUILLOU (a cura di), La Civiltà bizantina, oggetti e messaggio: architettura e ambiente di vita, Roma 1993, p. 42. 


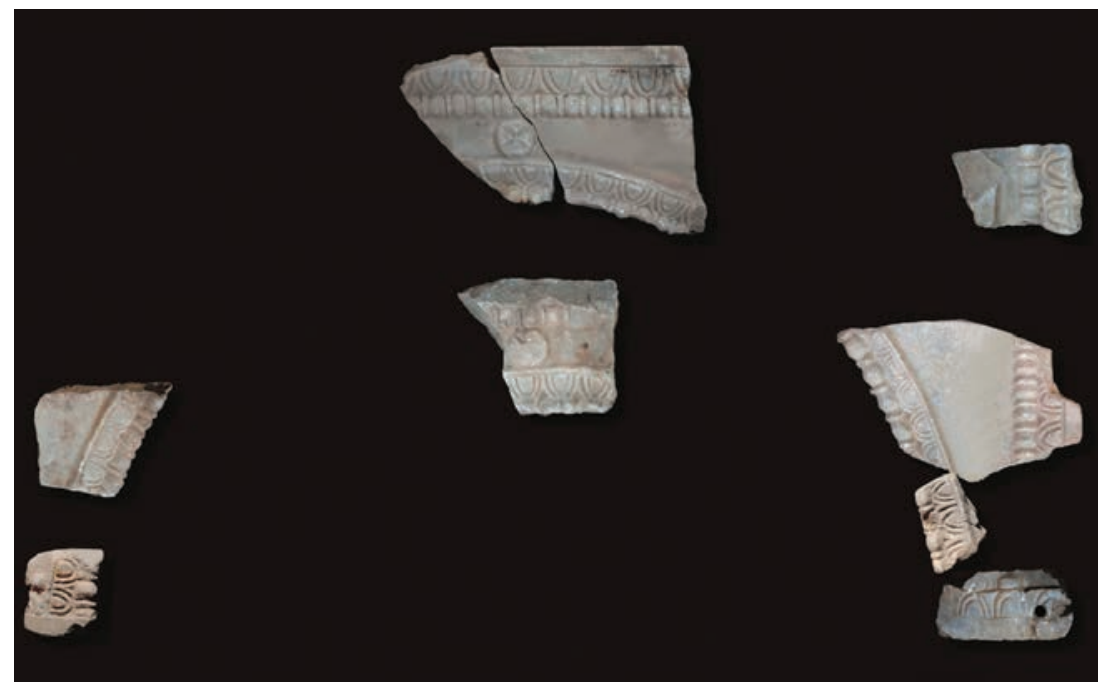

Fig. 8: i frammenti superstiti del ciborio della chiesa dell'acropoli (foto ed elaborazione di Diego Peirano)

“...Nel prezioso centro del lungo atrio si trova una fontana molto ampia, scavata dalla cima di Iaso, da cui un flusso sonoro zampilla e lancia nell'aria un getto che balza con forza dalla bronzea cannella..."24.

Secondo la Diegesis (26, p. 103) l'acqua sgorgava anche dalle bocche di 12 leoni (anch'essi presumibilmente di bronzo) posti in altrettante nicchie sul bordo della conca ${ }^{25}$, che doveva essere eccezionale sia per la ingegnosità della realizzazione, sia per le dimensioni, soprattutto avendo presente il ridotto formato dei labra e dei bacili rinvenuti a Iasos.

Inoltre:

Descript. S. Sophiae 629-632:

“... marmi scintillanti con venature ondulate, quanti ne producono le valli profonde delle vette di Iaso, marmi che mostrano striature oblique, di un rosso sangue e di un bianco livido..."
Nello stesso periodo, il marmo iasio è in opera in altri edifici di culto legati all'evergetismo dell'imperatore o del suo entourage: le chiese dei Santi Apostoli a Costantinopoli ${ }^{26}$, di S. Vitale ${ }^{27}$ e di S. Andrea Maggiore a Ravenna ${ }^{28}$, di S. Giovanni a Efeso $^{29}$, di S. Sergio a Gaza ${ }^{30}$, la chiesa "cattedrale" di Xanthos ${ }^{31}$ e la basilica di Gortyna/Metropolis ${ }^{32}$.

Se non sembra errato avanzare l'ipotesi che allora la proprietà delle cave fosse imperiale, l'amministrazione doveva nutrire comunque un interesse cogente per esse; di conseguenza anche nelle cave del marmo iasio operavano intermediari e artefici di provenienza costantinopolitana.

Potrebbe essere legata a ciò una ripresa dell'importazione, nella città, di altri marmi: che si tratti di materiale nuovo e non di riuso sembra avvalorato dall'assenza di tracce di rilavorazione su elementi architettonici e di arredo in edifici nei quali il riuso è attestato.

Rappresentano al meglio ciò, a Iasos, due chiese, l'una, la basilica dell'acropoli, costruita tra la fine del V e l'inizio del VI secolo, l'altra, la basilica dell'agorà, realizzata in età giustinianea33. Nella prima (fig. 4/3), la basilica dell'acropoli sono in marmo grigio, probabilmente cario, i plutei, uno dei quali riprende il tema ben noto nella produzione di VI secolo dei tralci d'uva e del pavone. Anche il ciborio appare un lavoro locale per il kyma ionico associato a un tondino che alterna fusaiole e perline ${ }^{34}$ (fig. 8): il motivo, diffuso nella Caria del VI secolo, lo è principalmente negli amboni ${ }^{35}$. Dell'ambone -del tipo egeo-costantinopolitano- della basilica restano la piattaforma, di marmo grigio scuro, $\mathrm{i}$ parapetti delle scalette (in marmo grigio con sottili venature brune) e, dello stesso marmo, quelle che sembrano parti del coronamento superiore (fig. 9) ${ }^{36}$.

\footnotetext{
${ }^{24}$ Questo e il passo seguente sono nella traduzione di M. L. FOBELLI, Un tempio per Giustiniano. Santa Sofia di Costantinopoli e la descrizione di Paolo Silenziario, Roma 2005, p. 71 e 73.

${ }^{25}$ M. L. FOBELLI, op. cit., p 148. Come non pensare, leggendo il passo, alla fontana della Grande Lavra del Monte Athos, di XI secolo (L. BOURAS, Some observations on the Grand Lavra Phiale at Mount Athos and its Bronze Strobilion, in $\Delta \varepsilon \lambda \tau \iota$ XAE 8, 1976, p. 85-96)? Sul marmo iasio in Santa Sofia cfr. anche A. GUIGLIA GUIDOBALDI, C. BARSANTI, Santa Sofia di Costantinopoli. L'arredo marmoreo della grande chiesa giustinianea, Città del Vaticano 2004, p. 67, 495, 497, 500, 555 ed E. RUSSO, La decorazione di Isidoro il Giovane per S. Sofia di Costantinopoli, Roma 2011, p. 103 e 107. Sui sectilia con marmo iasio a Saraçhane R. M. HARRISON, Excavations at Saraçhane in Istanbul, 1, Princeton University Press, 1986, p. 179 e passim.

${ }^{6}$ J. P. SODINI, Marble and Stoneworking in Byzantium, Seventh- Fifteenth Centuries, 2002, in E. LAIOU (ed.), The Economic History of Byzantium, Washington DC, 2002, p. 131

${ }_{27}^{27}$. NOVARA, Il rivestimento parietale nella chiesa di San Vitale di Ravenna: archeologia e antiquaria, in Ravenna Studi e Ricerche, 5/2, 1998, p. 61-117.

${ }^{28}$ M. MAZZOTTI, La basilica ravennate di Sant'Andrea Maggiore, in Corsi d'Arte Ravennate e Bizantina 1, 1959, p. 163 (dalla visita pastorale del 1605 apprendiamo che gli stipiti della porta centrale erano di marmo rosso) e $168 \mathrm{~s}$.

${ }_{29}^{2}$ Dove sono di marmo iasio i blocchi dello stilobate e le basi di colonna che recingono il presbiterio: F. W. DEICHMANN, op.cit. (n. 15), p. 216; J. P. SODINI, C. BARSANTI, A. GUIGLIA GUIDOBALDI, La sculpture architecturale en marbre au VI siècle à Costantinople et dans les régions sous l'influence costantinopolitaine, in Acta XIII Congressus internationalis Archaeologiae Christianae, (Split-Poreç, 29.9-1.10. 1994), Città del Vaticano, 1998, II, p. 315; J. P. SODINI, Marble and Stoneworking... op. cit. (n. 26), p. 133.

$3^{30}$ In posizione imprecisata: CORICIO, Laudatio Marciani, I, 17 ss.

${ }^{31}$ M. G. FROIDEVAUX, M. P. RAYNAUD, op. cit. (n. 21), p. 145.

${ }^{32}$ Nella corsia che incontra in direzione nord-sud la solea. Cfr. R. FARIOLI CAMPANATI, M. BOURBOUDAKIS, La decorazione pavimentale e parietale della cattedrale di Gortyna (Creta), Nuovi elementi di datazione del pavimento musivo della navata, in H. MORLIER (dir.), La Mosaïque Gréco-Romaine IX, 1, 2005 (Collection de l'Ecole Française de Rome, 352), p. 167, fig. 5a.

33 U. SERIN, Early Christian And Byzantine Churches At Iasos In Caria, Città del Vaticano 2004, p. 25-104 e 105-159; D. PEIRANO, Ricerche su assetti, arredi, percezione nella basilica dell'agora di Iasos, in Bollettino dell'Associazione Iasos di Caria 17, 2011, p. 10-15.

${ }^{34}$ Idem, Il ciborio della basilica dell'acropoli, in Boll. Iasos 18, 2012, p. 25-30.

35 Idem, Un frammento d'ambone inedito di tipo cario, in Boll. Iasos 20, 2014, p. 28.

${ }^{36}$ D. Peirano, E. GARberoglio, Amboni iasii: una rilettura con due inediti, in Iasos in età bizantina. Miscellanea di studi, Atti dell' Accademia delle Scienze di Ferrara 88, 2011, p. 339-344.
} 


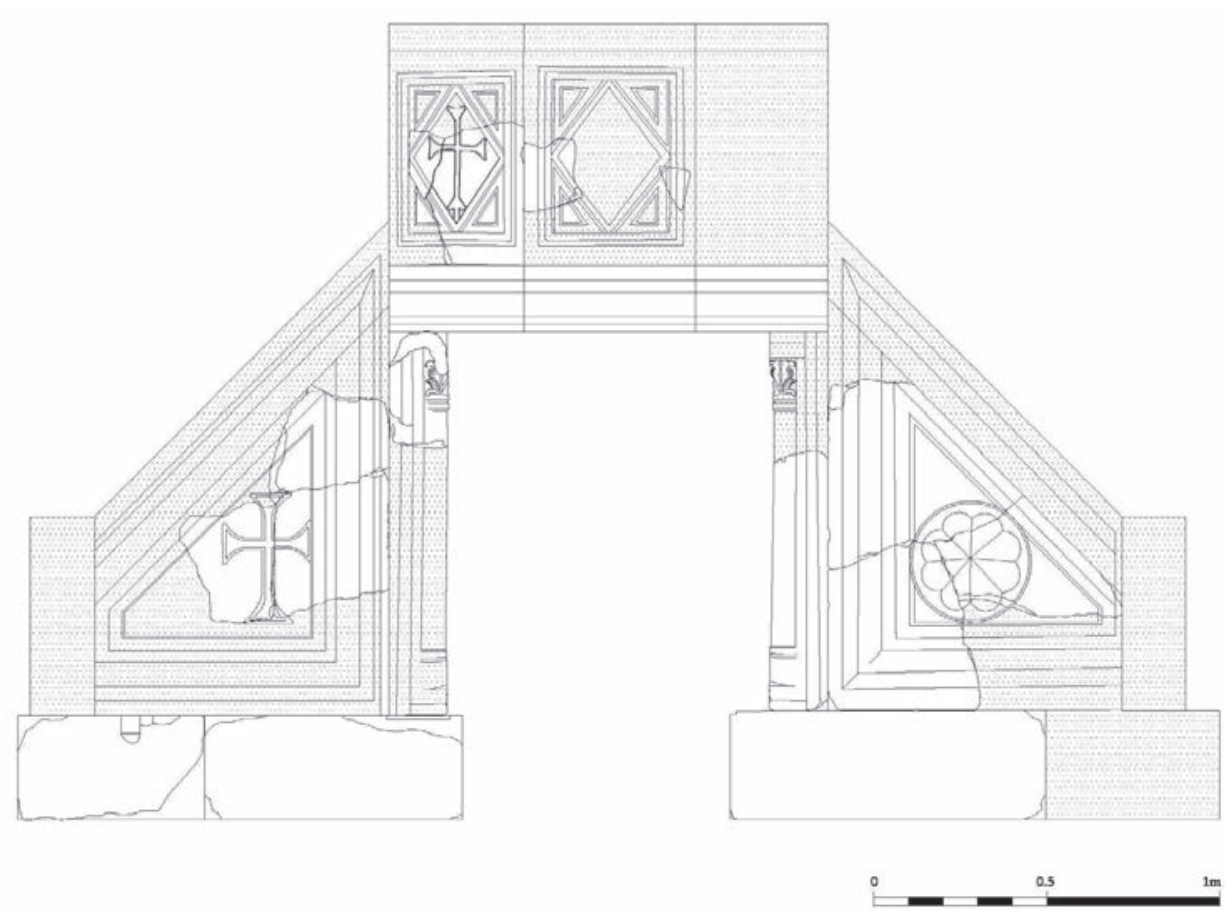

Fig. 9: ricostruzione -con retino sulle parti perdute-dell'ambone della chiesa dell'acropoli (disegno di Diego Peirano).

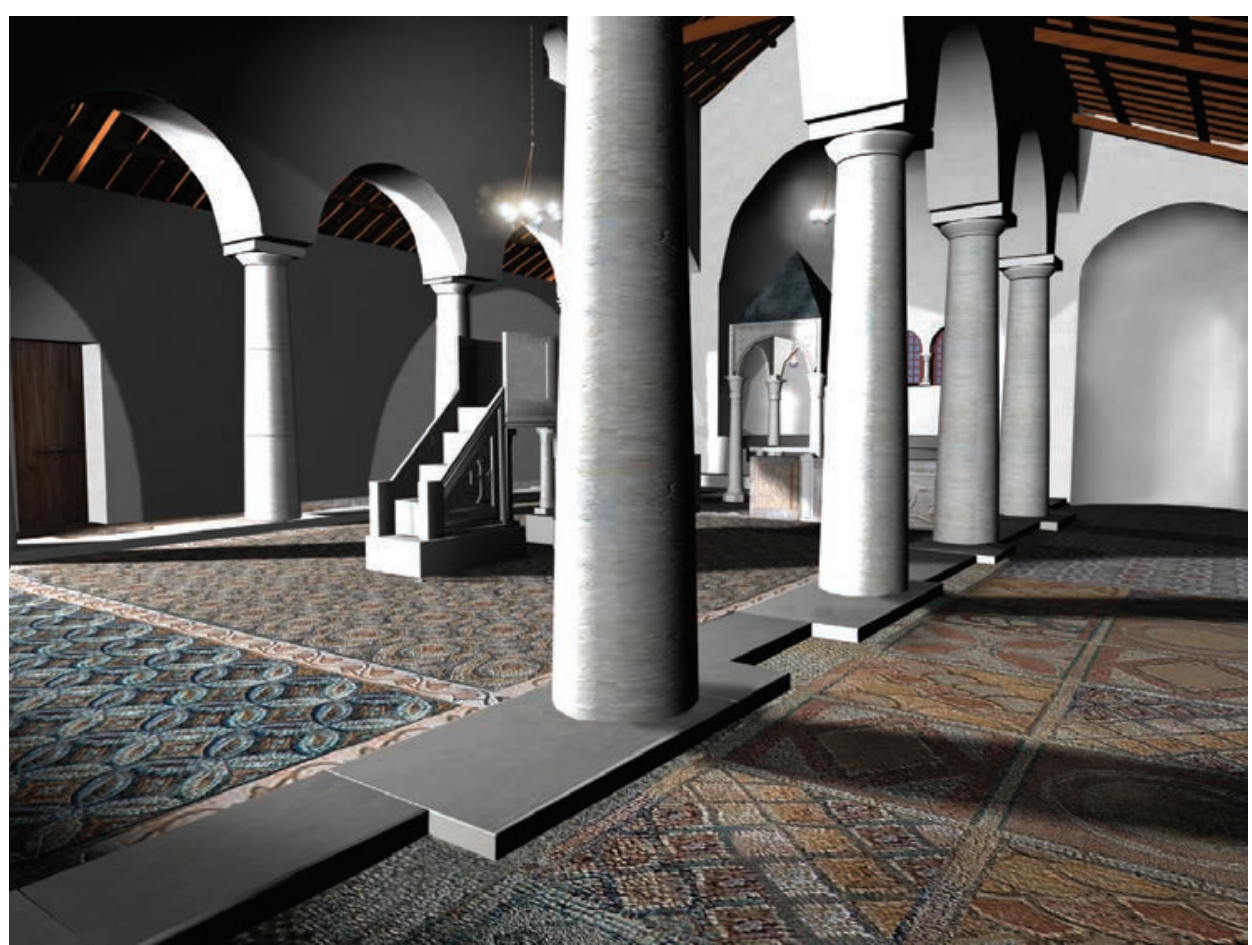

Fig. 10: ricostruzione della prima fase della basilica dell'acropoli (disegno di Diego Peirano)

accresciuti sia dalla lavorazione, sia dalla dislocazione dei diversi elementi. Il rosso iasio (cipollino, monocromo) si alterna al marmo bianco nelle piastrelle dei sectilia della navata centrale e dell'annesso settentrionale del nartece (fig. 11). Anche qui compare una piccola colonna di breccia rossa reimpiegata nel muro della cappella costruita in periodo medio-bizantino nella navata centrale (fig. 12), mentre di marmo grigio sono le basi e la cornice che chiudeva l'abside e di marmo bianco venato di grigio sono le colonne e gli stilobati. Il marmo grigio-bianco con venature ondulate e sottili striature brune compare nelle doppie colonne delle finestre, nei capitelli d'imposta che spartivano le navate e nelle mensole che reggevano il tetto. L'unico pluteo conservato è di marmo bianco, come le cornici parietali a foglie d'acanto che, per la rigida lavorazione, sembrano una realizzazione regionale (fig. 13). Anche l'ambone, indubbiamente un'opera "caria” per le scalette monolitiche e la piattaforma su arcate e pilastrini (fig. 14), fa uso di marmo grigio nella base e di marmo con venature ondulate e sottili striature brune nella piattaforma.

Alcune transenne lavorate a giorno con motivi diffusi in ambito costantinopolitano sono invece di fine marmo bianco zuccherino (fig. 15).

In altre parole, grazie agli scambi conseguenti alla diffusione del marmo locale, in età giustinianea sembra che si manifesti a Iasos una forte attrattiva per la varietà, gli effetti di luce e il colore derivanti dall'impiego di marmi di tipo diverso (fig. 16): alcuni, al pari degli schemi decorativi, provenivano dalla capitale.

Commercio e uso limitato: in considerazione dell'apprezzamento che riscuoteva, il marmo rosso rimaneva un prodotto di cui disporre raramente nelle imprese edili locali, risultando più vantaggioso esportarlo e acquistare altri marmi piuttosto che usarlo, sebbene

L'interno della chiesa dell'acropoli era quindi modulato su diverse tonalità di marmo grigio (fig. 10) e, se si escludono le tessere rosse dei mosaici pavimentali e una colonnetta di marmo rosso (della quale non si conosce l'originaria ubicazione), il marmo locale compare marginalmente, quasi che, all'alba del VI secolo, fosse destinato precipuamente all'esportazione.

Nella basilica dell'agora (fig. 4/4), invece, l'uso del marmo iasio si affianca a quello di altre pietre, con effetti cromatici proprio allora entrasse nella quotidianità degli iasei con una produzione minore di mense, tavole, bacili e mortai (fig. 17) che probabilmente si protrasse anche nel VII secolo ${ }^{37}$.

Non sappiamo datare la fine della coltivazione delle cave: considerando la circolazione locale della moneta come riflesso dei fenomeni storico-commerciali del lungo periodo, si constata come questa s'interrompa dopo la metà del VII secolo, riprenda con Leone VI (886-912) e prosegua fino a Teodoro I $(1205 / 8-1224)^{38}$.

${ }^{37}$ F. BERTI, D. PEIRANO, Il marmo iasio e alcuni tra i manufatti ad esso collegati, in Marmora 10, 2014, p. 25-37.

${ }^{38} \mathrm{~F}$. DELRIEUX, La circulation monétaire à Iasos durant la période byzantine, d'Anastase Ier Dikoros à la quatrième croisade, in Iasos in età bizantina. Miscellanea di studi, in Atti dell'Accademia delle Scienze di Ferrara, 88, 2012, p. 383- 426. 


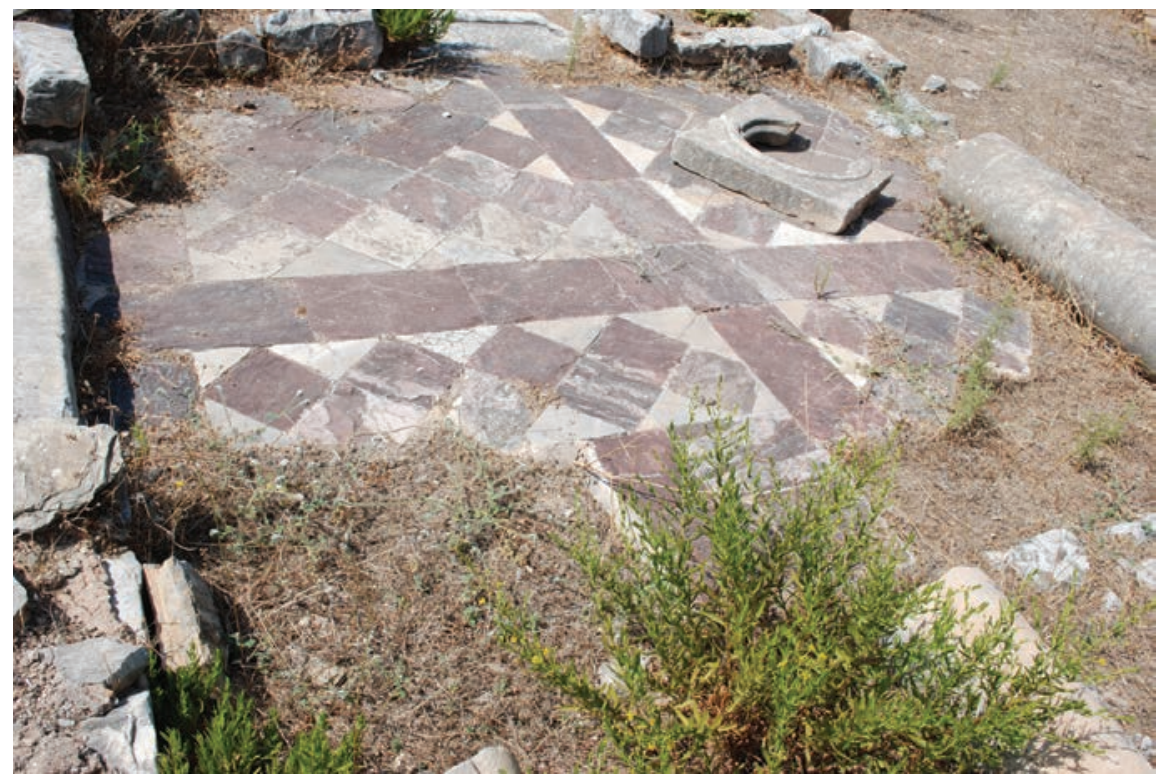

Fig. 11: l'annesso nord del nartece della basilica dell'agorà, pavimentato con un opus sectile fatto di lastre alternate di marmo iasio e bianco (foto Diego Peirano)

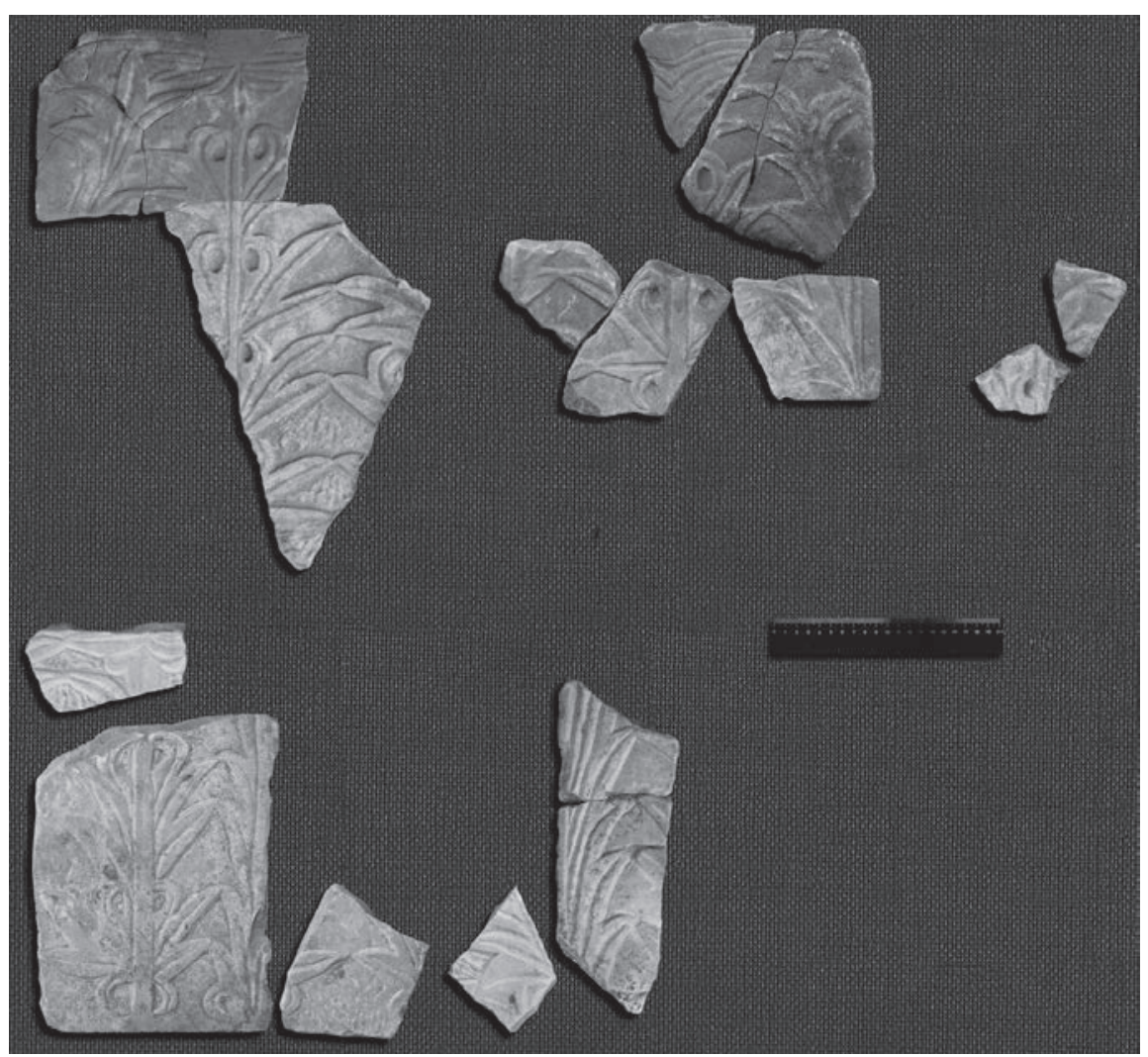

Fig. 13: cornici parietali a foglie d'acanto dalla basilica dell'agorà (foto ed elaborazione di Diego Peirano)

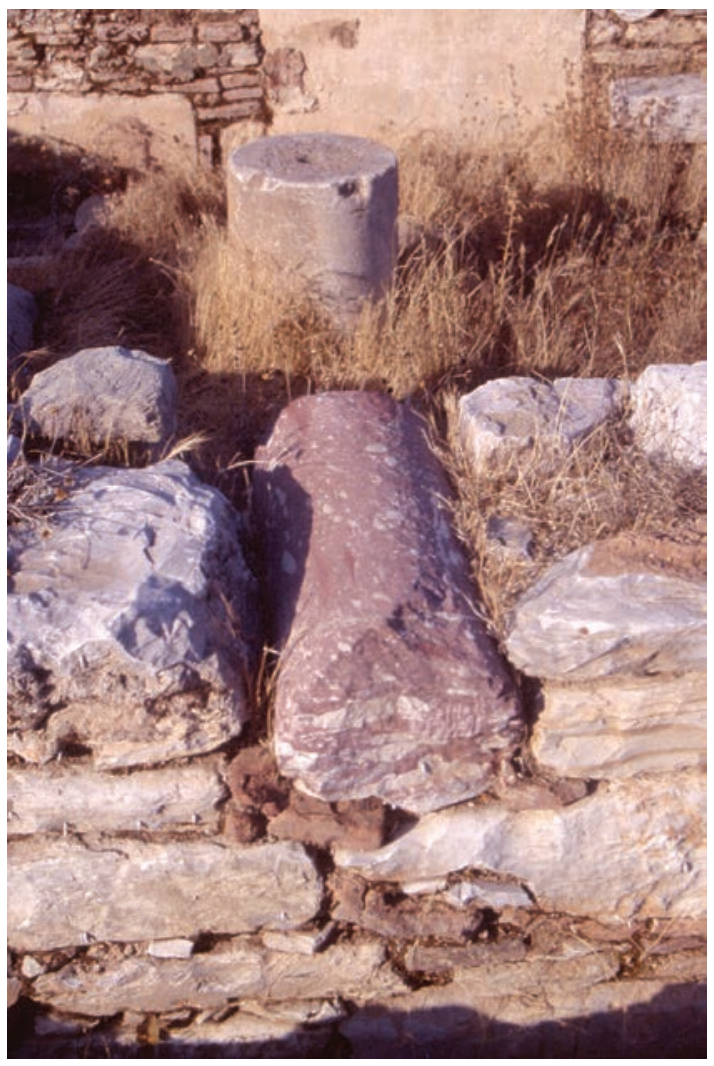

Fig. 12: colonna di breccia rossa reimpiegata nella cappella costruita in età medio-bizantina nella basilica dell'agorà (foto Daniela Baldoni)

Certo è che la presenza del marmo iasio a Costantinopoli va oltre il VI secolo, senza che ciò significhi necessariamente il perdurare dell'attività estrattiva: nella prima metà del IX secolo esso figura nel Palazzo di Teofilo ${ }^{39}$, poi nel kainourgion di Basilio ${ }^{40}$, nella seconda metà dell' XI nel monastero di Satyros ${ }^{41}$, in età comnena nella chiesa della Pammakaristos e di San Salvatore in Chora ${ }^{42}$.

Il conflittuale rapporto tra fisco e privati nei secoli IV-VII, il "marble-style" che si diffuse tra V e VI secolo, l'uso di spolia che non escluse la ricerca di nuovi marmi sono argomenti assai dibattuti ${ }^{43}$. Di fronte ad alcuni casi si è indotti a ritenere che nel IX secolo vi sia stata una ripresa delle lavorazioni e un incremento delle esportazioni: i materiali (ad esempio di XI secolo) e la circolazione di marmi cavati espressamente tuttavia sembrerebbero muoversi di pari passo

\footnotetext{
39 R. JANIN, Constantinople Byzantine. Développement urbain et répertoire topographique, Paris 1964, p. 89 (portico fatto costruire da Maurizio nel 587 e che probabilmente prese il nome dal marmo usato) e 114 (..le Carianos était le triomphe du marbre de Carie..); A. GUIGLIA, Il marmo a Costantinopoli dall'età dei Macedoni ai Paleologi, in C. BARSANTI, A. GUIGLIA, A. PARIBENI, Le officine dellimperatore....op. cit. (n.18), p. 139: opus sectile con marmo cario (829-842; fonti); J. M. FEATHERSTONE, Luxury in the Palace: the Buildings of Theophilus, in Istanbul Araștirmaları Ylllğı 2, 2013, passim e p. 37, fig. 5. $4^{\circ} \mathrm{La}$ sala aveva una cornice circolare in marmo iasio che racchiudeva l'immagine di un pavone: C. A. MANGO, The Art Of The Byzantine Empire 312-1453, Toronto 1986, p.192-193.

${ }^{41}$ A. RICCI, Archeologia urbana a Istanbul: il Kücükyalı ArkeoPark, in Arkeoloji ve Sanat 139, 2012, fig. 11: opus sectile.

${ }^{42}$ A. GUIGLIA GUIDOBALDI, La decorazione pavimentale bizantina in età paleologa, in A. IACOBINI, M. DELLA VALLE (a cura di), L'arte di Bisanzio e l'Italia al tempo dei Paleologi 1261- 1453, in Milion 5, 1999, p. 325: si ha notizia di un riquadro composto da 4 lastre con venature che formavano un disegno a clessidra bordato da verde antico.

${ }^{43} \mathrm{~J}$. P. SODINI, Le commerce des marbres à l'époque protobyzantine, in Hommes et richesses dans l'Empire byzantin, I. IV-VII siècle. Réalités byzantines, Paris 1989, p. 169 s.; Idem, Marble and Stoneworking... op. cit. (n. 26), nota 25.
} 


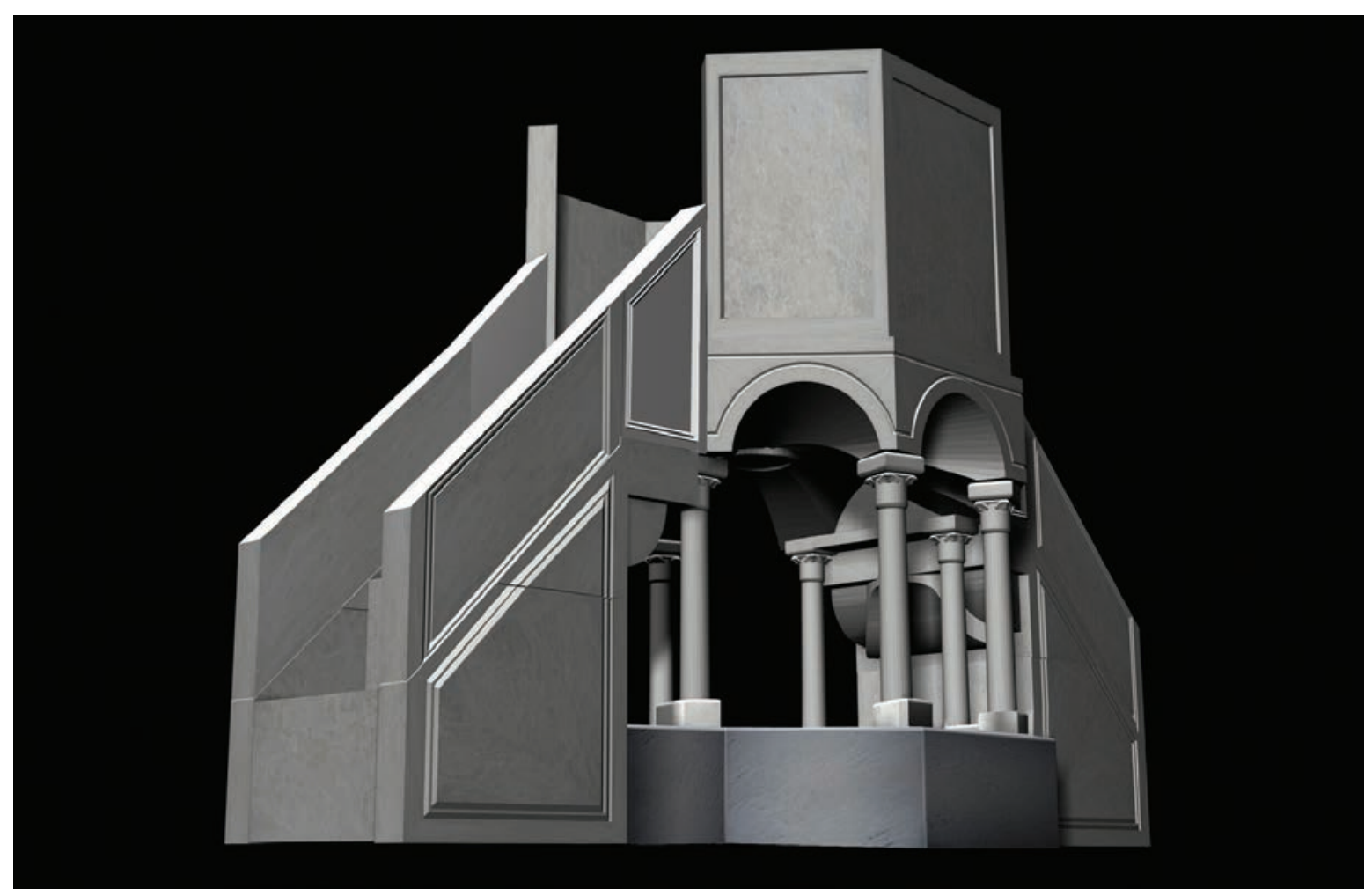

Fig. 14: ricostruzione dell'ambone della basilica dell'agorà (disegno di Diego Peirano)

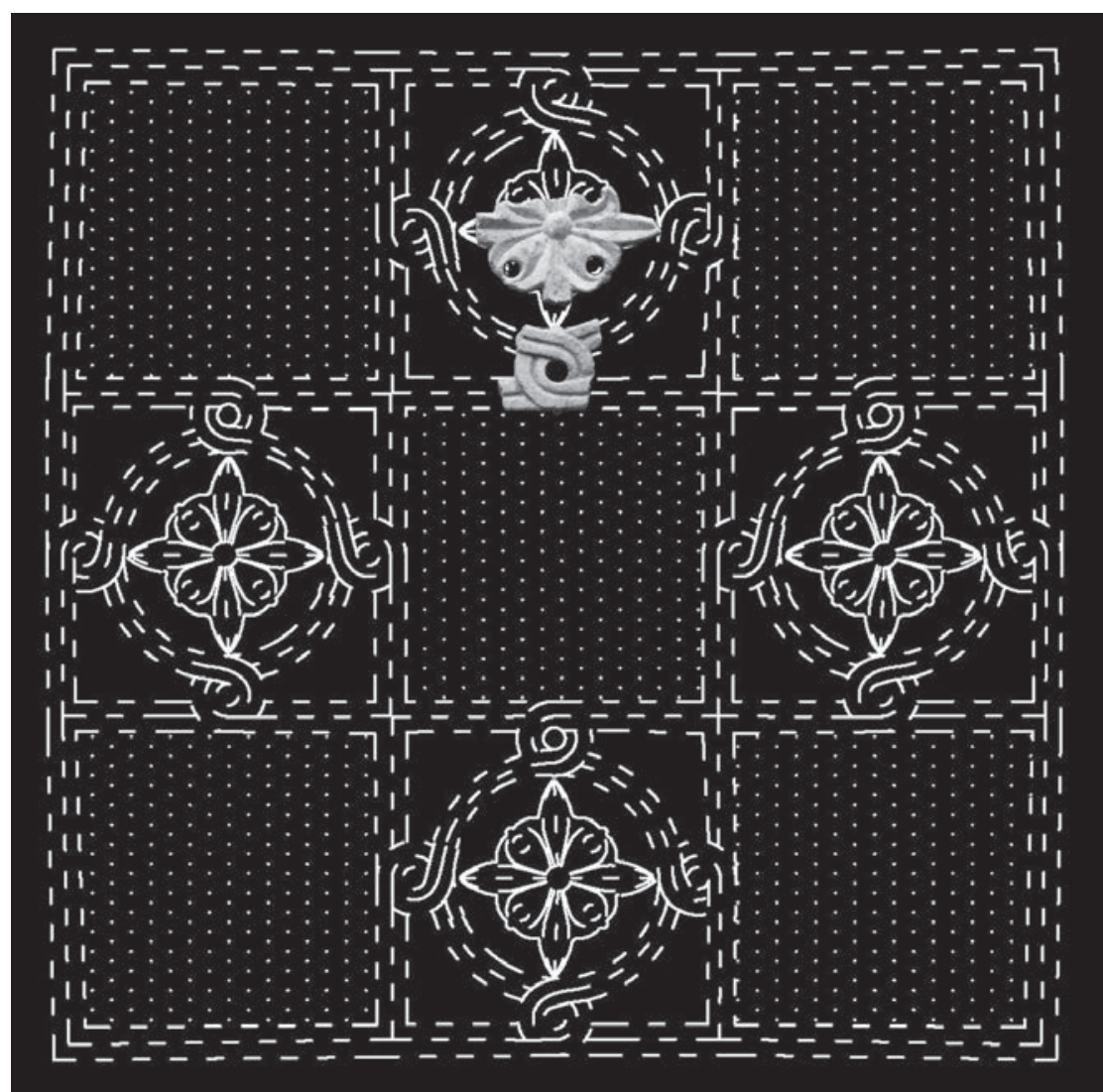

Fig. 15: ricostruzione di una transenna "a giorno" desunta dai frammenti rinvenuti nella basilica dell'agorà (disegno di Diego Peirano) con l'utilizzo delle “...ingenti scorte di blocchi di cava accumulatesi nel tempo presso le cave o in depositi"44.

Domande simili riguardano il marmo di Iasos impiegato negli edifici della capitale dopo il VI secolo: la pietra, anche a distanza di tempo, era fornita da depositi, dovunque essi fossero, oppure l'attività in cava continuò? E, in caso affermativo, fino a quando?

I più tardi nuclei dell'insediamento sulla penisola, profondamente mutato rispetto a quello che si era sviluppato in continuità con il tessuto urbanistico ellenistico e romano, possono suggerire qualche ipotesi?

La domanda ci induce a ritornare nel cantiere in cui si segava il marmo e al porto occidentale: se è difficile pensare che nel Balık Pazarı si sia lavorato a lungo, non va escluso che anche a distanza di tempo si prelevasse (onerosamente) quanto vi si trovava o vi era stato lasciato. La colonna sbozzata e alcuni blocchi di cipollino rosso nella chiesa costruita in età lascaride (giustappunto) sul porto occidentale 45 (fig. 4/5 e 18), parrebbero l'episodio conclusivo di questo processo: nell'edificio è sistematico il reimpiego di iscrizioni e di pezzi architettonici di ogni epoca, eppure la disponibilità della pietra locale non era ancora venuta meno sebbene mancasse oramai la capacità di lavorarla (il non-finito).

${ }_{44}$ C. BARSANTI, L'esportazione di marmi dal Proconneso nelle regioni pontiche durante il IV-VI secolo, in Rivista dell'Istituto Nazionale di Archeologia e Storia dell'Arte 12, 1989, p. 91-220; C. BARSANTI, A. GUIGLIA, A. PARIBENI, Le officine... op. cit. (n. 18), p. 118-125. Esemplare è il caso del marmo proconnesio: sulla sua diffusione sulle coste adriatiche in età giustinianea Y. A. MARANO, The Circulation of Marble in the Adriatic Sea at the Time of Justinian, che ringraziamo per aver reso disponibile un testo non ancora edito, e A. PARIBENI, Modalità e prassi operative delle maestranze del Proconneso nei cantieri bizantini tra Ve VI secolo, in O. Brandt et alii (a cura di), Acta XV congressus internationalis archaeologiae christianae (Toledo 8-12.9 2008), Città del Vaticano 2013, I, p. 1471-1478.

${ }^{45}$ U. SERIN, Some Observations on the middle Byzantine Church outside the east gate at Iasos, in R. PIEROBON BENOIT (a cura di), Iasos e la Caria. Nuovi studi e ricerche, in La Parola del Passato 6o, 2005, p. 156-178. 


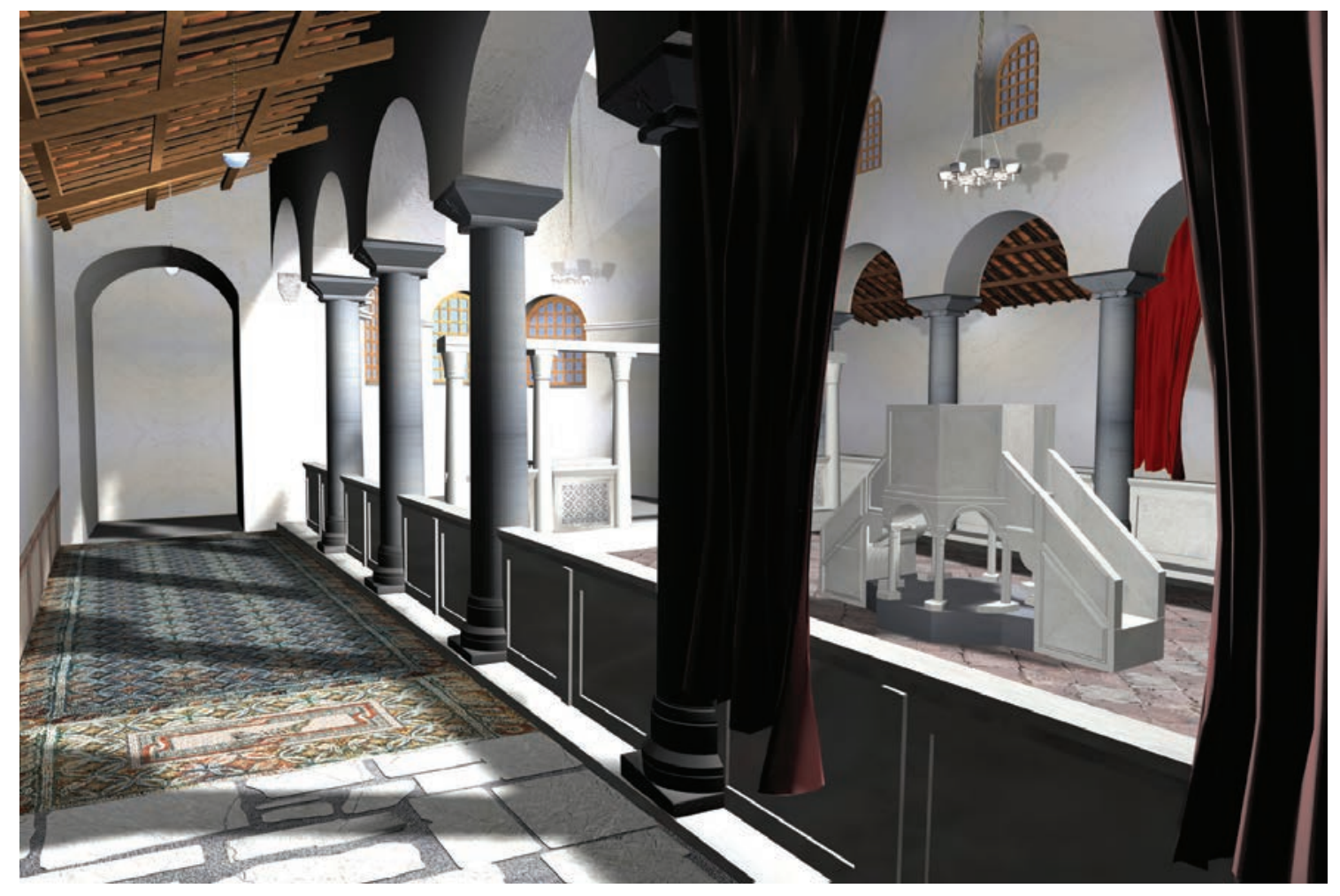

Fig. 16: ricostruzione della prima fase della basilica dell'agorà (disegno di Diego Peirano)
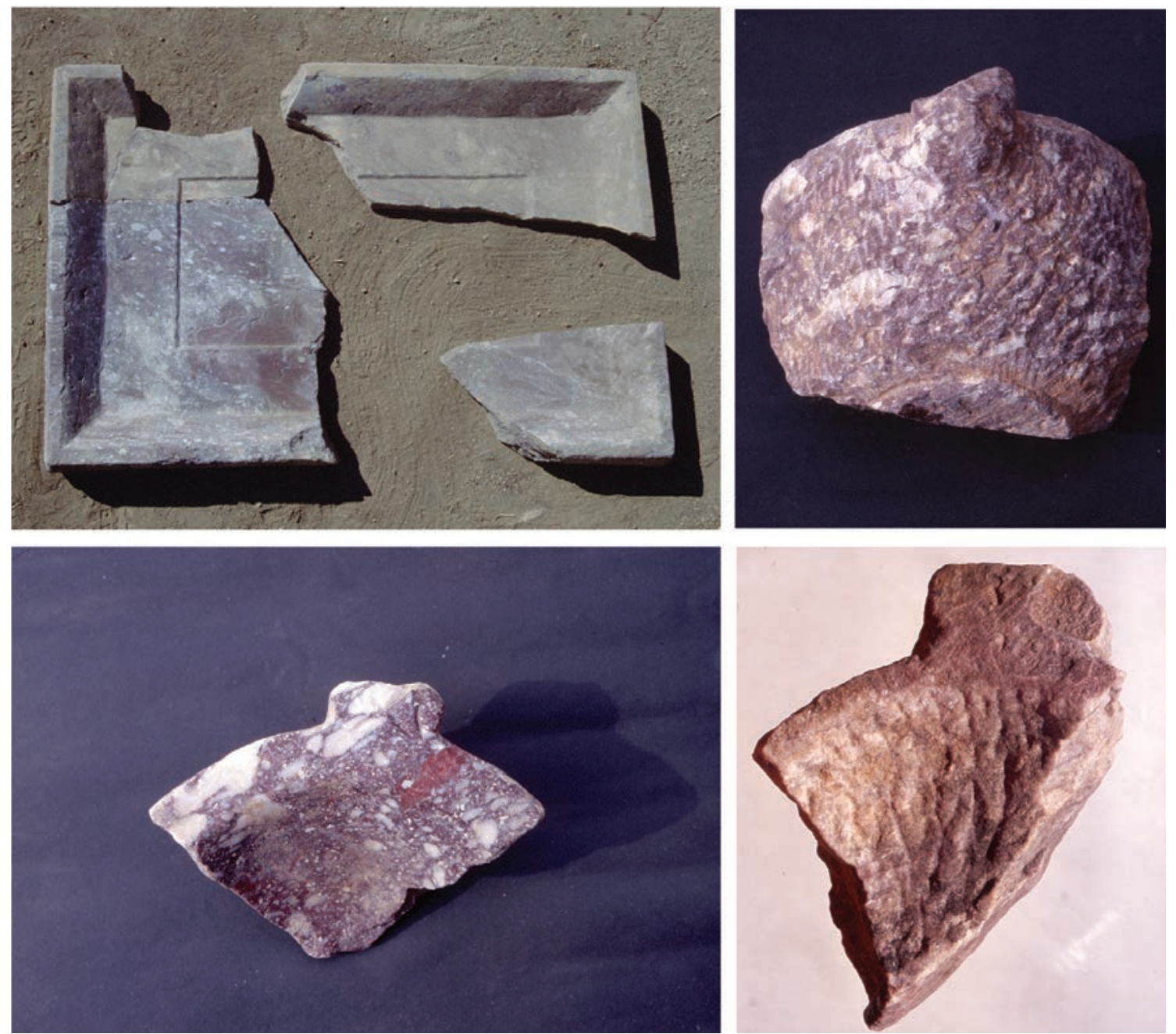

Fig. 17: in alto a sinistra una tavola rettangolare "clôturée", nelle altre immagini tre mortai con prese decorate e gradi diversi di lavorazione (foto Daniela Baldoni) 


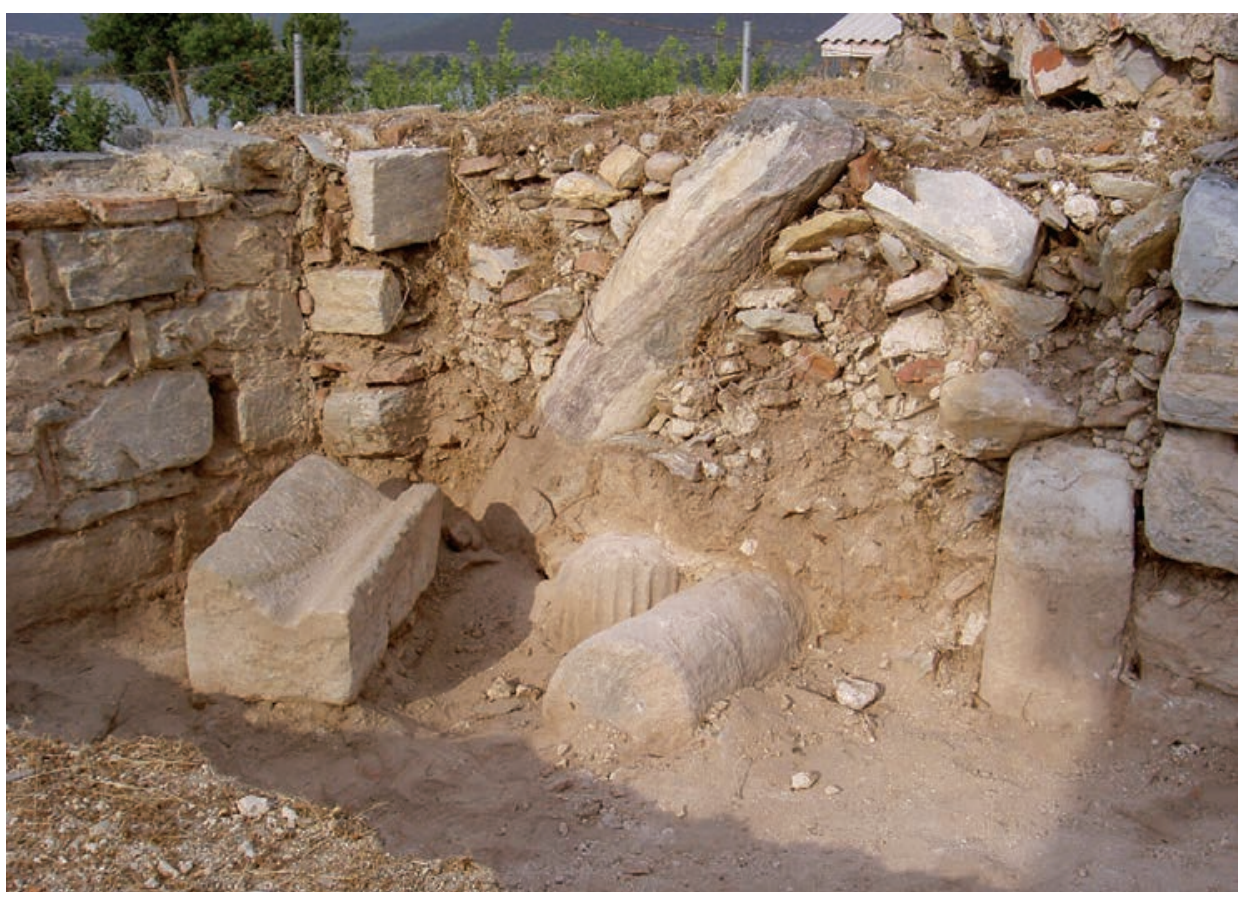

Fig. 18: la colonna in marmo cipollino rosso nell'abside settentrionale della chiesa lascaride (foto Daniela Baldoni)

Le entrate che derivavano ancora dal commercio di un materiale anche stoccato in loco potrebbero quindi spiegare (congiuntamente ad altri proventi) sia la ripresa di una parte del circolante dopo i raids arabi, quindi tra la fine del IX e il XIII secolo ${ }^{46}$, sia l'ingente quantità di vasellame "bizantino" e di anfore "bizantine" restituita dagli scavi della città ${ }^{47}$.

\footnotetext{
${ }^{46}$ Cfr. nota 38.

${ }^{47}$ E' frequente, ad esempio, l'anfora bollata di tipo 62 di Saraçhane J. W. HAYES, Excavations at Saraçhane in Istanbul, 2, The Pottery, Princeton 1992, p. 76 s., tav. 14,14), in circolazione tra il tardo XII secolo e gli inizi del secolo successivo.
} 